\title{
CAUCHY FRACTIONAL DERIVATIVE
}

\section{U. Kaya}

Bitlis Eren University, Bitlis, Turkey

E-mail:mat-ufuk@hotmail.com

In this paper, we introduce a new sort of fractional derivative. For this, we consider the Cauchy's integral formula for derivatives and modify it by using Laplace transform. So, we obtain the fractional derivative formula $F^{(\alpha)}(s)=L\{(-$ 1) $\left.{ }^{(\alpha)} L^{-1}\{F(s)\}\right\}$. Also, we find a relation between Weyl's fractional derivative and the formula above. Finally, we give some examples for fractional derivative of some elementary functions.

Keywords: Weyl's fractional derivative; fractional calculus; Laplace transform; Cauchy's integral formula for derivatives.

\section{Introduction}

Cauchy's integral formula for derivatives is given by the following relation

$$
F^{(n)}(s)=\frac{n !}{2 \pi i} \int_{C} \frac{F(w) d w}{(w-s)^{n+1}}, s \in \operatorname{int}(C), n \in \mathbb{N} .
$$

It calculates the derivative of order $n$ of an analytic function when $n$ is a nonnegative integer. Also, it seems to calculate the derivative of fractional order when we write $\alpha>0$ instead of $n$ :

$$
F^{(\alpha)}(s)=\frac{\Gamma(\alpha+1)}{2 \pi i} \int_{C} \frac{F(w) d w}{(w-s)^{\alpha+1}}, s \in \operatorname{int}(C) .
$$

However, it is not as simple as it looks. Because the contour integral in the formula (1) is so complicated. Moreover, the function $(w-s)^{\alpha+1}$ is multi-varied. Hence, the value of the contour integral in (1) is not independent of the choice of closed curve $C$. Formula (1) is an unpractical one to calculate the fractional derivative of a function. Therefore, it needs a modification. Here, we modify the formula (1). By making some calculations, we return it to the formula

$$
F^{(\alpha)}(s)=L\left\{(-x)^{\alpha} L^{-1}\{F\}\right\},
$$

where $L$ is the Laplace transform.

Weyl's fractional derivative is given by the following formula

$$
{ }_{s} W_{\infty}^{\alpha} F(s)=\frac{(-1)^{\alpha}}{\Gamma(n-\alpha)} \frac{d^{n}}{d s^{n}} \int_{s}^{\infty} \frac{F(t) d t}{(t-s)^{\alpha-n+1}},
$$

where $s>0, n-1<\alpha \leq n, n \in \mathbb{N}, \alpha>0$ [1]. Raina and Koul [1] proved in 1979 that the Laplace transform of the function $(-x)^{\alpha} f(x)$ is equal to $\alpha^{\text {th }}$ derivative, in the sense of Weyl, of the Laplace transform of $f$. This means that the fractional derivative of a function $F$ with the inverse Laplace transform can be calculated by the following formula

$$
{ }_{s} W_{\infty}^{\alpha} F(s)=L\left\{(-x)^{\alpha} L^{-1}\{F\}\right\} .
$$

In this work, we move the contour integral in the formula (1) to an infinite vertical line, and then we prove the relation (2). Finally, we give some examples.

\section{Laplace Transform and Cauchy's Integral Formula for Derivatives}

Let $f$ be a continuous function from $[0,+\infty)$ to $\mathbb{C}$ and satisfy the inequality $|f(x)| \leq M e^{a x}$ for some $a, M \in \mathbb{R}$. Then, its Laplace transform is defined by the following 


$$
L\{f\}=\int_{0}^{\infty} f(x) e^{-s x} d x,
$$

where $\operatorname{Re}(s)>a$ and $a \in \mathbb{R}$. We denote the Laplace transform of a function $f$ by $F(s)$, i.e.

$$
F(s)=\int_{0}^{\infty} f(x) e^{-s x} d x
$$

For example, the Laplace transform of the function $f(x)=(-x)^{\alpha} e^{w x}$ is

$$
F(s)=-\frac{\Gamma(\alpha+1)}{(w-s)^{\alpha+1}},
$$

where $\alpha>0, \operatorname{Re}(s)>\operatorname{Re}(w)$ and $\Gamma$ is the gamma function given by

$$
\Gamma(z)=\int_{0}^{\infty} x^{z-1} e^{-x} d x
$$

Laplace transform forms an invertible linear operator. Mellin's inverse formula for Laplace operator is given by the line integral:

$$
f(x)=\frac{1}{2 \pi i} \int_{c-i \infty}^{c+i \infty} F(w) e^{w x} d w
$$

where $c$ is a suitable real constant [2].

Now, we recall Cauchy's integral formula for derivative. Assume that $D$ is a region in the Complex plane $\mathbb{C}, F$ is an analytic function in $D, s \in D, C$ is a curve satisfying the condition $s \in \operatorname{int}(C)$ and $n \in \mathbb{N}$; then the $n^{\text {th }}$ derivative of $F$ is given by the following formula [3]

$$
F^{(n)}(s)=\frac{n !}{2 \pi i} \int_{C} \frac{F(w) d w}{(w-s)^{n+1}} .
$$

\section{Cauchy's Integral Formula for Derivatives on an Infinite Vertical Line}

Lemma 1. Assume that $F$ and $f$ are two functions satisfying the condition (5). Then, $F$ is an analytic function in the region $\operatorname{Re}(s)>a$ and it is bounded in the region $\operatorname{Re}(s) \geq c$ for each $c>a$. Besides, the improper integral in (5) is uniformly convergent on the region $\operatorname{Re}(s) \geq c$ [2].

Definition 1. If a function $F$ is the Laplace transform of a function, then we state that it is a Laplace type function.

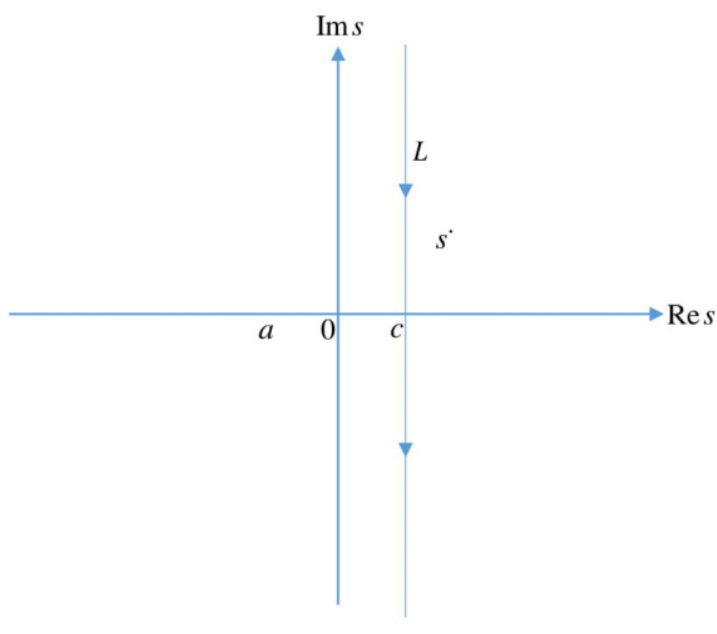

Fig. 1. Infinite vertical line

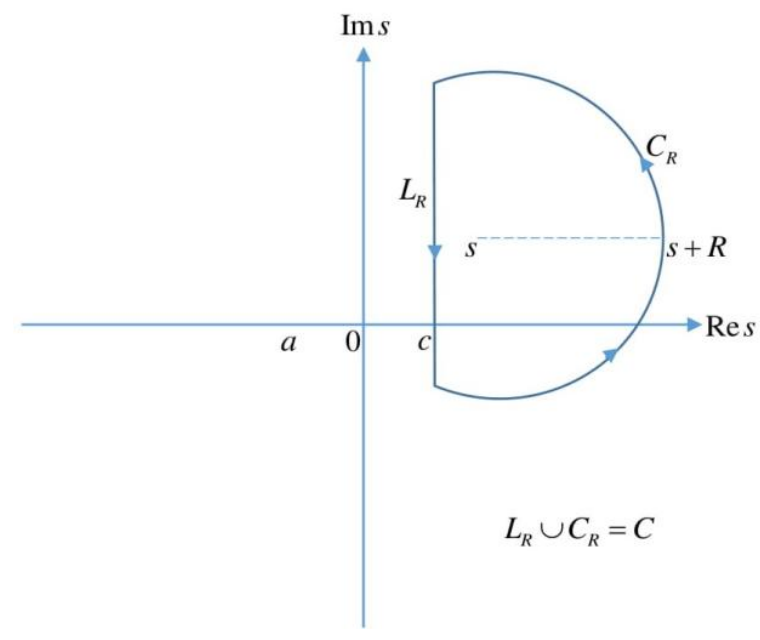

Fig. 2. Half circle 


\section{Математика}

Lemma 2. Let $F$ be a Laplace type function and $n \in \mathbb{N}$. Then, Cauchy's integral formula for derivatives (see (8)) can be written as follows:

$$
F^{(n)}(s)=\frac{n !}{2 \pi i} \int_{c+i \infty \infty}^{c-i \infty} \frac{F(w) d w}{(w-s)^{n+1}},
$$

where $a<c<\operatorname{Re}(s)$. Note that the integral above is taken on the infinite vertical line $L$ shown in Figure 1 .

Proof. By Cauchy's integral formula for derivatives (8) and by the structure of the curve $C$ shown in the Figure 2, we obtain

$$
F^{(n)}(s)=\frac{n !}{2 \pi i} \int_{C} \frac{F(w) d w}{(w-s)^{n+1}}=\frac{n !}{2 \pi i} \int_{L_{R}} \frac{F(w) d w}{(w-s)^{n+1}}+\frac{n !}{2 \pi i} \int_{C_{R}} \frac{F(w) d w}{(w-s)^{n+1}} .
$$

By Lemma 1, there exists a positive number $M$ such that $|F(w)| \leq M$. Then, we have

$$
\left|\frac{n !}{2 \pi i} \int_{C_{R}} \frac{F(w) d w}{(w-s)^{n+1}}\right| \leq \frac{n !}{2 \pi} \int_{C_{R}} \frac{|F(w)||d w|}{|w-s|^{n+1}} \leq \frac{M n !}{2 \pi R^{n+1}} \int_{C_{R}}|d w|=\frac{M n !}{2 R^{n}} .
$$

The last relation shows that the value of the integral on the half circle $C_{R}$ vanishes when $R \rightarrow+\infty$. This ends the proof.

\section{Cauchy Fractional Derivative}

Definition 2. Let $F$ be a Laplace type function and $\alpha$ be a positive real number. We denote Cauchy fractional derivative of order $\alpha$ by ${ }_{s} C_{\infty}^{\alpha} F(s)$ and define as the following relation

$$
{ }_{s} C_{\infty}^{\alpha} F(s)=\frac{\Gamma(\alpha+1)}{2 \pi i} \int_{c+i \infty}^{c-i \infty} \frac{F(w) d w}{(w-s)^{\alpha+1}},
$$

where $a<c<\operatorname{Re}(s)$ (see Fig. 1).

Remark. Since the function $F$ in the formula (9) is a Laplace type function, then there exists at least one real number $a$ such that $F$ is analytic in the region $\operatorname{Re}(w)>a$. Furthermore, the function $(w-s)^{\alpha+1}$ has

analytic branches in the region

$$
\inf \{k \in \mathbb{N} \mid k \alpha \in \mathbb{N}\}
$$

$$
\mathbb{C} \backslash\{w=\xi+i \operatorname{Im}(s) \mid \xi \geq \operatorname{Re}(s)\} .
$$

So, there are $\inf \{k \in \mathbb{N} \mid k \alpha \in \mathbb{N}\}$ values of Cauchy fractional derivative for any function $F$. And also, one can simply see that these values are independent of the choice of the real number $c$ in the interval $(a, \operatorname{Re}(s))$.

Theorem 1. Let $F$ be a Laplace type function and $\alpha$ be a positive real number. Then the Cauchy fractional derivative ${ }_{s} C_{\infty}^{\alpha} F(s)$ holds the following relation

$$
{ }_{s} C_{\infty}^{\alpha} F(s)=L\left\{(-x)^{\alpha} L^{-1}\{F\}\right\}={ }_{s} W_{\infty}^{\alpha} F(s) .
$$

Proof. We begin by writing the definition of the Cauchy fractional derivative:

By the formula (6), we obtain

$$
{ }_{s} C_{\infty}^{\alpha} F(s)=\frac{1}{2 \pi i} \int_{c+i \infty}^{c-i \infty} F(w) \frac{\Gamma(\alpha+1)}{(w-s)^{\alpha+1}} d w .
$$

$$
{ }_{s} C_{\infty}^{\alpha} F(s)=-\frac{1}{2 \pi i} \int_{c+i \infty}^{c-i \infty} F(w) L\left\{(-x)^{\alpha} e^{w x}\right\} d w=\frac{1}{2 \pi i} \int_{c-i \infty}^{c+i \infty} F(w)\left(\int_{0}^{\infty}(-x)^{\alpha} e^{(w-s) x} d x\right) d w .
$$


By using the uniform convergence of the Laplace's improper integral and the boundedness of the function $F$ (see Lemma 1), we can change the order of integration, i.e.

$$
{ }_{s} C_{\infty}^{\alpha} F(s)=\frac{1}{2 \pi i} \int_{0}^{\infty c+i \infty} \int_{c-i \infty}^{\infty} F(w)(-x)^{\alpha} e^{(w-s) x} d w d x=\int_{0}^{\infty}(-x)^{\alpha} e^{-s x}\left(\frac{1}{2 \pi i} \int_{c-i \infty}^{c+i \infty} F(w) e^{w x} d w\right) d x .
$$

By the inverse Laplace formula (7), the fractional derivative ${ }_{s} C_{\infty}^{\alpha} F(s)$ can be written as

$$
{ }_{s} C_{\infty}^{\alpha} F(s)=\int_{0}^{\infty}(-x)^{\alpha} e^{-s x} L^{-1}\{F\} d x=L\left\{(-x)^{\alpha} L^{-1}\{F\}\right\} .
$$

The formulas (4) and (10) completes the proof.

Example 1. By using well-known formula

we have

$$
L\left\{x^{r-1}\right\}=\frac{\Gamma(r)}{s^{r}}, r>0,
$$

$$
{ }_{s} C_{\infty}^{\alpha} \frac{1}{s^{r}}=L\left\{(-x)^{\alpha} L^{-1}\left\{\frac{1}{s^{r}}\right\}\right\}=\frac{1}{\Gamma(r)} L\left\{(-x)^{\alpha} x^{r-1}\right\}=\frac{(-1)^{\alpha}}{\Gamma(r)} L\left\{x^{\alpha+r-1}\right\}=\frac{(-1)^{\alpha} \Gamma(\alpha+r)}{\Gamma(r) s^{\alpha+r}} .
$$

In a similar way, we can obtain the following

where $a \in \mathbb{C}$.

$$
{ }_{s} C_{\infty}^{\alpha} \frac{1}{(s-a)^{r}}=\frac{(-1)^{\alpha} \Gamma(\alpha+r)}{\Gamma(r)(s-a)^{\alpha+r}},
$$

A table of some functions' $\frac{1}{2}^{\text {th }}$ derivatives is given in the following:

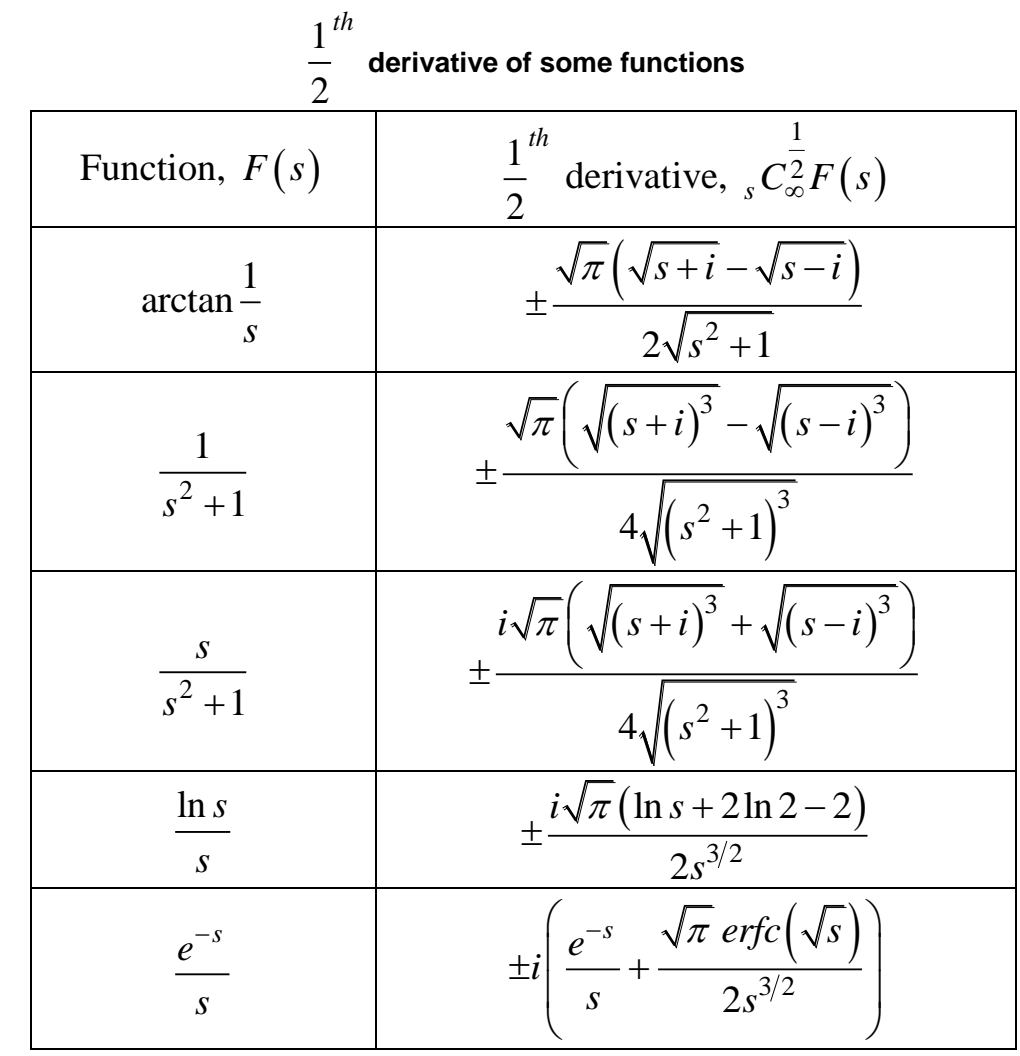




\section{Математика}

Example 2. We can use the Dirac Delta function, which is a generalized function denoted by $\delta(x)$ and defined by the equality $\int_{-\infty}^{\infty} \delta(x-b) f(x)=f(b)$, to calculate the Cauchy fractional derivative. Let $F(s)=e^{-b s}, b>0$. Then, the inverse Laplace transform of it is $\delta(x-b)$ and we have

$$
{ }_{s} W_{\infty}^{\alpha} e^{-b s}=L\left\{(-x)^{\alpha} L^{-1}\left\{e^{-b s}\right\}\right\}=L\left\{(-x)^{\alpha} \delta(x-b)\right\}=(-b)^{\alpha} e^{-b s} \text {. }
$$

To illustrate,

$$
{ }_{s} W_{\infty}^{\frac{1}{2}} e^{-s}= \pm i e^{-s}
$$

\section{References}

1. Raina R.K., Koul C.L. On Weyl Fractional Calculus. Proc. Amer. Math. Soc., 1979, Vol. 73, no. 2, pp. 188-192. DOI: 10.1090/S0002-9939-1979-0516462-4

2. Debnath L., Bhatta D. Integral Transforms and Their Applications. CRC press, Boca Raton, 2014,818 p.

3. Ahlfors V.L. Complex Analysis. McGraw-Hill Inc., New York, 1979, 336 p.

Received September 4, 2020

Bulletin of the South Ural State University Series "Mathematics. Mechanics. Physics" 2020, vol. 12, no. 4, pp. 28-32

DOI: 10.14529/mmph200403

\section{ДРОБНАЯ ПРОИЗВОДНАЯ КОШИ}

\section{У. Кайя \\ Университет Битлис Эрен, Битлис, Турция \\ E-mail:mat-ufuk@hotmail.com}

Вводится новый вид дробной производной. Рассматривая интегральную формулу Коши для производных, и модифицируя её с помощью преобразования Лапласа, автор получает формулу дробной производной в виде $F^{(\alpha)}(s)=L\left\{(-1)^{(\alpha)} L^{-1}\{F(s)\}\right\}$. Установлена связь между дробной производной Вейля и приведенной выше формулой. В завершение работы приведены примеры дробных производных некоторых элементарных функций.

Ключевые слова: дробная прочзводная Вейля; дробное исчисление; преобразование Лапласа; интегральная формула Коши для производных.

\section{Литература}

1. Raina, R.K. On Weyl Fractional Calculus / R.K. Raina, C.L. Koul // Proc. Amer. Math. Soc. 1979. - Vol. 73, no. 2. - P. 188-192.

2. Debnath, L. Integral Transforms and Their Applications / L. Debnath, D. Bhatta. - CRC press, Boca Raton, 2014. - 818 p.

3. Ahlfors, V.L. Complex Analysis / V.L. Ahlfors. - McGraw-Hill Inc., New York, 1979. - 336 p.

Поступила в редакцию 4 сентября 2020 г. 